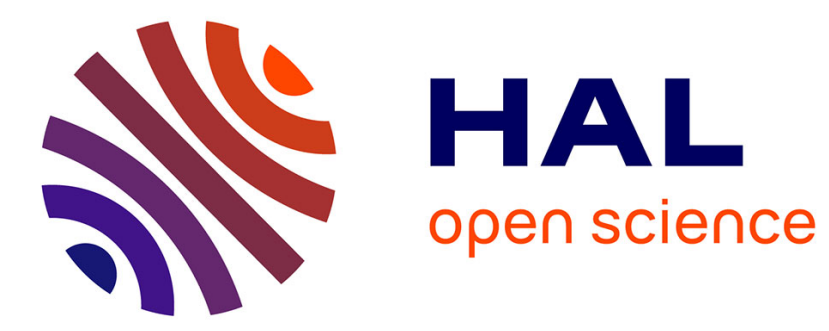

\title{
A metric for evaluating density level of wireless sensor networks
}

\author{
Ahmed Akl, Thierry Gayraud, Pascal Berthou
}

\section{To cite this version:}

Ahmed Akl, Thierry Gayraud, Pascal Berthou. A metric for evaluating density level of wireless sensor networks. Wireless Days (WD), Oct 2011, Niagara Falls, Canada. pp.1-3. hal-00661015

\section{HAL Id: hal-00661015 https://hal.science/hal-00661015}

Submitted on 18 Jan 2012

HAL is a multi-disciplinary open access archive for the deposit and dissemination of scientific research documents, whether they are published or not. The documents may come from teaching and research institutions in France or abroad, or from public or private research centers.
L'archive ouverte pluridisciplinaire HAL, est destinée au dépôt et à la diffusion de documents scientifiques de niveau recherche, publiés ou non, émanant des établissements d'enseignement et de recherche français ou étrangers, des laboratoires publics ou privés. 


\title{
A Metric for Evaluating Density Level of Wireless Sensor Networks
}

\author{
Ahmed AKL, Thierry GAYRAUD, and Pascal BERTHOU \\ CNRS-LAAS, 7 Avenue Colonel Roche, F-31077 Toulouse, France \\ Université de Toulouse; UPS, INSA, INP, ISAE; LAAS; F-31077 Toulouse, France \\ Toulouse, France \\ (aakl, gayraud, berthou)@laas.fr
}

\begin{abstract}
The term "Dense networks" is usually used to identify networks having large number of nodes within a small area while affecting network performance. Starting from this concept, different networking solutions were proposed. However, it seems to us that this view is very abstract and needs to be further investigated. Knowing the network density level can be of great importance in determining the network parameters. In this paper, we firstly examine the effect of network size over its performance, then introduce a metric to determine if a network is considered as a "dense network" or not. Finally, we applied our metric on experimental results published in the CRAWDAD dataset site to verify our metric.
\end{abstract}

Keywords- Sensor networks, Dense networks)

\section{INTRODUCTION}

Wireless Sensor Networks (WSNs) are a special type of adhoc networks where its simplest form consists of a set of sensors for data capturing, usually environmental data (i.e., temperature), and sending them to a base station through a heterogeneous network, which may contain base stations, different types of sensors, actuators, and processing elements [1]. The nodes (i.e., sensors) are characterized by being limited in resources (i.e., power, storage, processing, etc...), and small in size. They are powered by tiny batteries where the battery power is usually directly proportional to its size. Such power constraint has a great effect over the node activities. The major power consuming activities are mobility and communication. However, power consumption due to mobility is not a must; a WSN can be static or the nodes attached to a moving object, so that no power is consumed through mobility. On the contrary, power consumption due to communication is a must to perform the required task. A single hop communication can be more power consuming than a multiple hop communication [2] because more transmission power is required.

Apparently, a dense network can raise different problems to MAC layer such as overhearing, communication grouping, over-provisioning, and neighbor state [4]. Moreover, the negative effect propagates to the Network [5],[6] and Application [3], [7] layers where it can't be over-passed.

Section II gives an overview on dense networks, showing the factors affecting density, and how density is usually calculated. In section III, we discuss our proposed metric.
Section IV shows the simulation held by NS2 simulator. Section $\mathrm{V}$ shows the usage of the proposed metric to analyze a real experimentation results. Finally, we introduce the conclusion and future work.

\section{DENSE NETWORKS: AN OVERVIEW}

The term Dense Network is usually used in the ad-hoc networking context to represent high concentration of nodes within certain area. There are alternative terms (i.e., massively dense networks, and very large networks), other than "Dense networks", used in the context, but the term "Dense Networks" is the most used one. However, this term can be misleading because it gives the impression that the population around all nodes is high, although it may be true for only some of them [8].

In a dense ad-hoc network, too many communication links are sometimes detrimental for energy consumption, network throughput, and quality of service. In spite of the imposed difficulties, some WSN techniques prefer the usage of a dense network to obtain better performance since it encourages the cooperation between sensors.

\section{A. Factors affecting density}

Different factors have influence over the network density; some of them are controllable while the others are not. However, it is difficult to consider some factors as being absolutely controllable or uncontrollable (i.e., Mobility). The main factors are mobility, obstacles, transmission range and deployment scheme.

\section{B. Calculating network density}

Toumpis [8] surveyed different wireless networks solutions based on analogies with physics. He noted that, most of these solutions are based on the assumption that the network has high node density. However, few attempts were done to find a measure for density. Bulusu [9] proposed an equation to calculate the network density and many other publications had used it[10], [11]. He said that Network Density $(\mu)$ can be roughly calculated as $\mu=\frac{\left(N \pi R^{2}\right)}{A}$; Where $\mathrm{N}$ is the number of nodes in area $\mathrm{A}$, and $\mathrm{R}$ is the radio transmission range for a 
disk communication model. A more precise equation was proposed by Toumpis [9] who defined the node density as $d(r)=\lim _{|A| \longrightarrow 0} \frac{N(A)}{|A|}$, where density is measured as the number of nodes per $\mathrm{m}^{2}$.

These equations are derived from the idea that network density is the amount of certain quantity (i.e., number of nodes) within certain area, and this quantity is only dependent on the node transmission range. However, it seems to us that such assumption is very abstract, since there are other factors (i.e., mobility, obstacles, etc...), which are not considered in the equation and can affect the number of connected nodes. For example, a node can have 5 neighbors because they are located within its transmission range even if some of them are not really connected due to their short transmission range.

\section{A METRIC FOR EVALUATING NETWORK DENSITY}

Although different solutions are based on the assumption of having a dense network, there are still some questions that need further investigations; Does the number of nodes per unit area is the only required measure to identify a dense network?

According to the studied use cases, we can deduce that: (1) The number of nodes within the transmission range is not an enough measure for network density; (2) The minimum number of nodes to achieve a dense network depends on different factors in addition to the number of neighboring nodes and (3) it may be necessary to have different degrees of density not only dense and non-dense.

For these reasons, we propose the usage of Effective Density $(E D)$ as an indication of the density level. The $E D$ of a node is the ratio between the number of single hop connected nodes $(N)$, and the node Throughput $(t h)$, where $E D=N / t h$. In other words, we can consider $E D$ as a measure to find out how much each additional node is going to participate in changing the Throughput

When more nodes enter the transmission range of a node, the node connectivity increases causing th to increase, so that the enhancement of Throughput decreases the effect of increasing neighbors over the effective density. In other words, the increase of Throughput suppresses the effect of increasing $\mathrm{N}$.

\section{Simulation RESUlts}

The objective of this simulation is, firstly, to study the effect of increasing the number of nodes over the receiver Throughput, secondly, to show the Effective Density and how it is changed with respect to nodes number, and throughput.

The configuration of the simulated scenario is as follows; the network terrain is $500 \mathrm{~m} \times 500 \mathrm{~m}$ with the Tx and Rx nodes located at $(0,0)$ and $(500,500)$, respectively. Node transmission range is adjusted, so that the Tx and Rx are not directly connected, but they are connected through a group of single hop connected neighbors. The simulation is repeated 9 times where 10 randomly distributed nodes are added to the network each time. CBR connection of $0.3 \mathrm{Mb}$ is used to inject data traffic to the network, and throughput was calculated at the application Layer level. The simulation duration is 600sec.

Fig. 1 shows the change of Effective Density with respect to the number of nodes. It is noticeable that the rate of change had increased after adding 40 nodes. This means that the network became crowded, so each additional node causes more

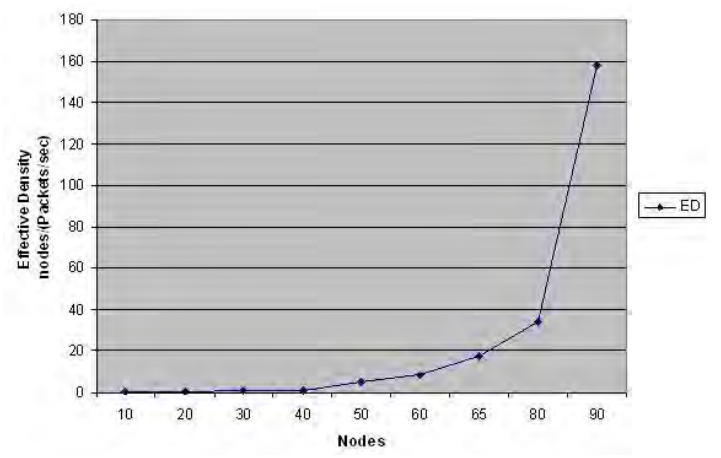

Fig. 1: Effective Density

collisions and Throughput degradation becomes obvious. However, there is still one more question, what is the value after which the network is considered dense. In fact, there is not a discrete value; each protocol and application should determine its own threshold values. For example, an application can consider the previous network as non-dense until it has 40 nodes, then semi-dense until 80 nodes, and finally highly dense for more than 80 nodes. On the other hand, another application can consider it as non-dense till 80 nodes and highly dense after that.

\section{EXPERIMENTAL ANALYSIS}

CRAWDAD [12] provides rich datasets originating from different real experimentations in various networking domains; one of them was conducted by Liu [13]. He used a test bed to have a practical outdoor comparison between different ad-hoc protocols. The experimentation was held in an area of $225 \mathrm{mx} 365 \mathrm{~m}$ where 40 laptops were moving randomly. They were divided into groups, of 10 laptops each, where each group was randomly distributed into one of the 4 zones in the terrain. The moving pattern is as follows; each device randomly chooses a location in a different zone and moves straight toward it, then the process is repeated after reaching the destination. GPS location data and traffic data are located for each device

We used the dataset generated by Liu [13] to show the effect of changing the number of neighboring nodes over Throughput. As nodes move, the number of direct connected neighbors changes, and is calculated through the SIN values, so that inactive nodes are excluded from the calculations.

Fig. 2 shows the values of Effective Density (ED), Throughput, and number of nodes $(N)$ as they change with time. It is noticeable that Throughput has the same pattern as the change of number of nodes. This is because almost all network parameters are kept constant and it is the number of neighboring nodes that change due to mobility.

In Zone1, $E D$ started at its maximum, because $N$ was high and Throughput was very low. This means that the large 
number of nodes didn't enhance Throughput. However, ED decreased dramatically with decreasing $N$ because Throughput didn't change too much. This indicates that at this period, the current distribution of high number of nodes didn't increase connectivity, but it affected Throughput negatively; this can be noticed in the trivial change of Throughput when compared to the large drop in number of nodes. Consequently, ED decreased quickly as $N$ decreases.

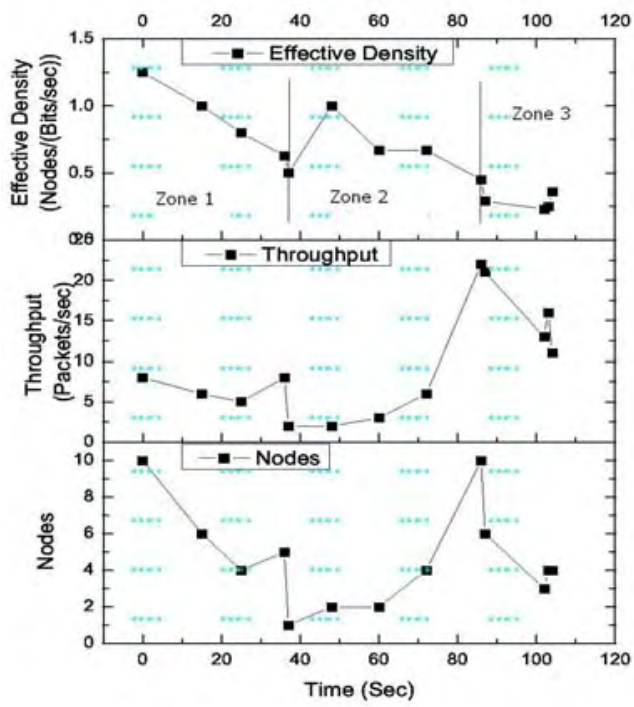

Fig. 2: Effective Density, Throughput, Nodes Vs time

In Zone2, the rate of change of nodes is almost the same as the rate of change of Throughput, so there were no great changes in the $E D$. This means that when $E D$ is constant, any change in $\mathrm{N}$ is accompanied with the same proportional change in throughput. In other words, the new injected nodes are affecting the network positively and causing an increase in Throughput.

Zone3 has the same effect as Zone2, but in the opposite sense. $E D$ is almost constant due to, approximately, the same decreasing rate of both $N$ and Throughput

We deduce that $E D$ can be used as a measure for the effect of injected or leaving nodes over performance in terms of Throughput.

\section{CONCLUSION AND FUTURE WORK}

The network density is an important parameter for network description. However, we believe that the current definition of network density only represents the number of neighboring nodes within the node transmission range, and doesn't represent its effect over the network. In other words, the network density calculation, which is presented in the literature, is not an enough metric to judge the network state as being dense or non-dense since it doesn't consider the network performance. Thus, we propose the usage of Effective Density as a new measure, which allows us to study the dynamic effect of the neighbor's number. Moreover, it allows us to divide the network into zones of different densities where each zone can behave according to the influence of its current population.

We conducted a simulation as a proof of the concept where we showed how the Effective Density is influenced by the changing number of node's neighbors and its Throughput. Then, we showed the metric applicability over a data set extracted from a real experimentation.

Our future work is to integrate our metric within a protocol that uses network density as its control parameter to show how our metric can enhance the protocol behavior

\section{REFERENCES}

[1] F. Dressler, Self-Organization in Sensor and Actor Networks, 1st ed. WILEY Publishing, Inc., 2007, p. 386.

[2] J. Zhao and R. Govindan, "Understanding Packet Delivery Performance In Dense Wireless Sensor Networks," Proceedings of the 1st international conference on Embedded networked sensor systems, pp. 1-13, 2003.

[3] S. Cho, H. Shin, S. Han, H. Cha, and R. Ha, "Density-adaptive network reprogramming protocol for wireless sensor networks," Wireless Communication and Mobile Computing, no. 2009, pp. 857-874, 2010

[4] G. P. Halkes and K. G. Langendoen, "Crankshaft : An Energy-Efficient MAC-Protocol for Dense Wireless Sensor Networks," Proceedings of the 4th European conference on Wireless sensor networks, pp. 228-244, 2007.

[5] A. Silva, E. Altman, and G. Alfano, "Magnetworks : how mobility impacts the design of Mobile Networks," IEEE Proceedings INFOCOM 2010, pp. 1-9, 2010.

[6] D. Liarokapis, A. Shahrabi, A. Komninos, and A. Shahrabi, "DibA : An Adaptive Broadcasting Scheme in Mobile Ad Hoc Networks," Proceedings of the 6th International Conference on Mobile Technology, Application, pp. 224-231, 2009.

[7] M. Haghpanahi, M. Kalantari, and M. Shayman, "Implementing Information Paths in a Dense Wireless Sensor Network," IEEE Global Telecommunications Conference, GLOBECOM 2009, pp. 1-7, 2009.

[8] S. Toumpis, "A survey of recent results on wireless networks based on analogies with physics," Computer Networks, vol. 52, pp. 360-383, 2008

[9] N. Bulusu, D. Estrin, L. Girod, J. Heidemann, and U. S. C. Isi, "Scalable Coordination for Wireless Sensor Networks : Self-Configuring Localization Systems," Proceedings of the Sixth International Symposium on Communication Theory and Applications (ISCTA '01), no. July, pp. 1-6, 2001.

[10] S.-L. Wu and Y.-C. Tseng, Wireless Ad Hoc Networks (Personal-Area, Local-Area, and the Sensory-Area Networks), 1st ed. Auerbach publications, 2007, p. 664.

[11] I. F. Akyildiz, W. Su, Y. Sankarasubramaniam, and E. Cayirci, "A Survey on Sensor Networks," IEEE Communications Magazine, no. August, pp. 102-114, 2002.

[12] R. S. Gray et al., "CRAWDAD data set dartmouth/outdoor (v. 2006-1106)," $2006 . \quad$ [Online]. Available: http://crawdad.cs.dartmouth.edu/dartmouth/outdoor.

[13] J. Liu et al., "Empirical Validation of Wireless Models in Simulations of Ad Hoc Routing Protocols," Simulation Journal, vol. 81, pp. 307-323, 2005. 